Cahiers d'études italiennes

18 | 2014

NOVECENTO... E DINTORNI

Da Torino a Parigi: Laura Malvano storica e critica d'arte

\title{
Laura Malvano e Barnaba da Modena
}

Laura Malvano et Barnaba da Modena

Laura Malvano and Barnaba da Modena

Giovanni Romano

(2) OpenEdition

Journals

Edizione digitale

URL: http://journals.openedition.org/cei/1639

DOI: $10.4000 /$ cei. 1639

ISSN: 2260-779X

Editore

UGA Éditions/Université Grenoble Alpes

Edizione cartacea

Data di pubblicazione: 30 mars 2014

Paginazione: $95-106$

ISBN: 978-2-84310-268-4

ISSN: $1770-9571$

Notizia bibliografica digitale

Giovanni Romano, «Laura Malvano e Barnaba da Modena», Cahiers d'études italiennes [Online], $18 \mid$

2014, online dal 30 septembre 2015, consultato il 26 mars 2021. URL: http://journals.openedition.org/ cei/1639 ; DOI: https://doi.org/10.4000/cei.1639 


\title{
LAURA MALVANO E BARNABA DA MODENA
}

\author{
Giovanni Romano
}

Laura ha discusso la tesi di laurea all'Università di Torino l'8 novembre 1956, con primo relatore Anna Maria Brizio e controrelatore (allora si diceva così) Aldo Bertini; la Biblioteca del Dipartimento Discipline Artistiche dell'Università di Torino conserva la copia dell'elaborato utilizzata da Bertini (si riconosce la sua calligrafia nei pochi appunti segnati a margine) e il testo costituisce una monografia a tutto tondo su Barnaba da Modena, come nessuno finora è riuscito a realizzare ${ }^{I}$. L'indice corrisponde a come si intendeva il genere tesi mezzo secolo fa, in area purovisibilista e crociana: una prima parte dedicata allo «sviluppo della pittura di Barnaba da Modena» e una seconda (in cinque segmenti) di apparati giustificativi: "lineamenti per una storia della critica di Barnaba da Modena», "catalogo delle opere», "catalogo delle opere perdute», "catalogo delle opere attribuite» (tutti e tre i cataloghi sono condotti con molta cura), «regesti» ed «elenco bibliografico». Laura apparteneva ad una famiglia di pittori e di letterati e lo si avverte nell'aderenza delle letture critiche e nella grazia della scrittura, che non scivola mai in scimmiottature longhiane, a quel tempo non infrequenti. Credo che sotto questo aspetto il controllo della Brizio abbia agito da deterrente. Ne trascrivo due esempi, sintomatici del suo modo di accostarsi ai dipinti: nella Madonna di Barnaba alla Sabauda (I370) «la pittura leggera e morbida con cui è accarezzato il volto della Vergine o quella intensa e succosa che modella la tunichetta e il manto del Bambino, ci fanno sentire sempre viva la presenza di un pittore di polso" ${ }^{2}$; è letterariamente più colorita l'evocazione del concerto angelico intorno alla Vergine incoronata nell'anta sinistra del dittico di Londra (I374):

I. L. Malvano, Barnaba da Modena, tesi in Storia dell'Arte, relatore A. M. Brizio, Università degli Studi di Torino, Facoltà di Lettere, a. a. I955-1956. L’Archivio Storico dell'Università di Torino conserva un'altra copia della tesi (devo l'informazione alla cortesia di Paola Novaria). Il voto ottenuto fu di Io8 su iro e Laura un poco se ne vantava, come segno della sua scarsa sensibilità per una superlativa votazione accademica.

2. Ivi, p. 66. 
[...] l'angelo che in primo piano strimpella l'organo, spalancando la bocca in un potente «a solo» e intanto strabuzza gli occhi con aria adirata verso le iperboliche trombe soffiate con troppo zelo sul suo capo; i due angeli zampognari attenti e diligenti, e quello che suona i pifferi, curioso e distratto; e ancora nelle ultime file i due angeli che commentano tra di loro la scena con un'espressione divertita sui volti grassocci ${ }^{3}$.

I problemi che rendono difficile la definizione critica della personalità di Barnaba sono, ancora oggi, quelli che ha affrontato Laura all'inizio degli anni Cinquanta, ed è notevole che, in alcuni passaggi, il suo giudizio sulla cultura figurativa del pittore risulti di particolare sottigliezza e attualità. Nonostante la univoca definizione longhiana («intento alla poetica vitalesca»), appoggiata al problematico Noli me tangere della collezione Fila, Laura si sforza di articolare la formazione emiliana di Barnaba, al di là delle mascherature arcaizzanti (parla di «zelo reazionario») ${ }^{4}$, evocando i nomi di Simone dei Crocefissi, di Dalmasio, del creduto Jacopino di Francesco, per Longhi «cugino dei riminesi»`. Stupisce la giusta insistenza sui riminesi per spiegare gli arcaismi del maestro, quando i pittori di Rimini erano ancora personalità molto nebulose nel panorama del Trecento italiano e la cronologia della pittura bolognese si appoggiava a supporti molto fragili, messi poi radicalmente in discussione dalla scoperta della data I333 sul trittico già a Perpignan e ora tornato al Louvre (la data fu resa nota da Michel Laclotte solo nel 1976) ${ }^{6}$. Il ribaltamento della successione cronologica dei trecentisti bolognesi, a scapito della leadership di Vitale, coinvolge naturalmente la nostra possibilità di immaginare i primi passi di Barnaba, purtroppo non documentati, e di precisare la radice e i caratteri della sua evidente propensione retrospettiva. Barnaba deve essersi avviato in patria su quanto lasciato da Tomaso da Modena prima del passaggio a Treviso (I349), ma deve aver subito conosciuto e apprezzato anche il doppio registro stilistico della pittura bolognese, tra moderna sperimentazione naturalistica e confezione di icone secondo la maniera greca, come documentano il Maestro dei polittici bolognesi (Pseudo-Jacopino) e, a date più avanzate, Simone di Filippo «dei crocefissi» ${ }^{7}$. Laura sospetta in effetti

3. Ivi, pp. 68-69.

4. Ivi, p. 4.

5. Ivi, pp. 8-Io.

6. M. Laclotte e É. Mognetti, Avignon-Musée du Petit Palais. Peinture italienne, Parigi, Éditions des Musées nationaux, 1976, p. I00. La cronologia del primo Trecento bolognese era già stata messa in discussione da L. Bellosi, Buffalmacco e il Trionfo della Morte, Torino, Einaudi, 1974, pp. 83-87. Un accenno di sfuggita ai riminesi, che Laura non poteva conoscere, è in R. Longhi, Progetti di lavoro di Roberto Longhi «Genova pittrice», in «Paragone», $\mathrm{n}^{\circ}$ 349-35I, marzo-maggio 1979, p. II.

7. Sul «doppio registro stilistico» della pittura bolognese si vedano da ultimo gli interventi di Massimo Ferretti e Daniele Benati in M. Medica (a cura di), Giotto e Bologna, Cinisello Balsamo (Milano), Silvana, 20I0, pp. 5I-85. 
che per Barnaba si possa parlare di «separazione pratica di due distinte attività $[. .$.$] l'una destinata ad una produzione corrente e più mercantile$ [...] l'altra più eletta e forse di più scelta destinazione» ${ }^{8}$.

Porta oggi qualche luce sui primi anni del nostro pittore la Madonna con il Bambino nella Parrocchiale di Lerma (Alessandria) (fig. I), ufficialmente pubblicata solo nel 1997 (dalla Rossetti Brezzi), ma restaurata già nel 1973 presso la Soprintendenza di Torino (operatore Elvio Gamarra, sotto la mia direzione) ${ }^{9}$. Nonostante i danni subiti la Madonna di Lerma si impone su tutti gli altri esempi di Barnaba per solenne nobiltà di atteggiamento e di sguardo, e si stacca sul fondo d'oro, ora quasi completamente perduto, per un contorno di musicale eleganza e studiata asimmetria; i colori nell'abito del Bambino sono di gusto un poco esotico e squisitamente cangianti, ma quanto colpisce in special modo è il tipo fisico dei personaggi, di una morbida carnosità che evoca direttamente i modelli di Tomaso. È difficile proporre una datazione, ma credo che possiamo fiduciosamente regredire di non poco rispetto al I36I, prima data a noi nota per la presenza di Barnaba a Genova, cittadino genovese da qualche anno, con bottega ben assestata. Resta da sottolineare che nel caso di Lerma non si avverte quell'incupirsi delle ombreggiature che è tratto costante nella pittura di Barnaba e che ha indotto gli studiosi a confronti con esempi bizantini sempre difficilmente precisabili. Si trattò di un bizantinismo evocato genericamente, più di simulazione che di ricalco, che certo può indurre in qualche tentazione: al Museo Bizantino di Atene una predella con la Natività $n^{\circ}$ T255 e la Madonna dell'umiltà $n^{\circ}$ T277 adescano il visitatore con ingannevoli (?) caratteri liguri. Si apre a questo punto il problema della fortuna della pittura bizantina a Genova, documentata con certezza dagli affreschi della controfacciata in Duomo, ma che impone come tema di ricerca la presenza in Liguria di tavole "greche» a date alte, quindi utili anche per la storia di Barnaba: approdarono tardi in Liguria il dittico bizantino della Cattedrale di Cuenca, di provenienza genovese, e forse anche la Dormitio Virginis del santuario di Montallegro presso Chiavari ${ }^{\text {Io }}$, ma devono essere

8. L. Malvano, Barnaba da Modena, cit., p. 39.

9. E. Rossetti Brezzi, Testimonianze trecentesche nel territorio alessandrino, in G. Romano (a cura di), Pittura e miniatura del Trecento in Piemonte, Torino, Fondazione CRT, Banca CRT, 1997, pp. 30-3I; vedi anche G. Algeri, Nuove testimonianze per la pittura del Quattrocento in Liguria, in S. Marconi (a cura di), Scritti e immagini in onore di Corrado Maltese, con il coordinamento scientifico di M. Dalai Emiliani, Roma, Quasar, 1997, p. 445 e n. 3 a p. 449.

IO. E. Parma, Il dittico reliquiario bizantino dei despoti dell'Epiro donato da Giovanni Domenico Castagnola Spinola alla Cattedrale di Cuenca, in A. R. Calderoni Masetti, C. Di Fabio e M. Marcenaro (a cura di), Tessuti, oreficerie, miniatura in Liguria, XIII-XV secolo, Atti del convegno internazionale di studi, GenovaBordighera, 22-25 maggio 1997, Bordighera, Alzani, 1999, pp. 55-64; G. Algeri, Testimonianze d'arte nella 
esistiti a Genova casi analoghi alla Madonna dell'Uscetto nella Cattedrale di Alessandria o, meglio ancora, opere come la Madonna delle Grazie nella Cattedrale di Cambrai (di provenienza italiana), che ripete lo stesso tipo iconografico, ma che stilisticamente evoca i quesiti posti dal Maestro del dittico Sterbini a Palazzo Venezia (un maestro che si crede di gravitazione adriatica, ma che potrebbe essere stato attivo piuttosto sulle coste tirreniche) ${ }^{\text {II }}$. La perduta Madonna del pontile, in San Domenico a Genova, "opere graeco depicta, nuncupata la mora ${ }^{\mathrm{I} 2}$, era stata forse parte del tesoro costantinopolitano che papa Innocenzo III reclamò inutilmente dai "predoni» genovesi («iconas [...] cruces aureas [...] scyphos argenteos [...] capsellas») ${ }^{\mathrm{I}}$ ed è possibile che parte di queste meraviglie fosse ancora, nel Quattrocento, in mano di Eliano Spinola (del ramo di Luccoli): per fama giunta fino a Roma, a papa Paolo II, Eliano possedeva «immagines sanctorum operis antiqui ex Graecia allatae, quas illi iconas vocant; aulea item, textura acuve picta [...] vascula quoque cuiusque modi cari lapidis» ${ }^{\mathrm{I}}$.

diocesi di Chiavari. Opere restaurate 1982-1992, catalogo della mostra a Chiavari, Genova, Sagep, I993, pp. I3 e 19. Sull'insieme di questi problemi vedi ora C. Di FabIo, Bisanzio a Genova fra XII e XIV secolo. Documenti e memorie d'arte, in P. Boccardo e C. Di FABIo (a cura di), Genova e l'Europa mediterranea. Opere, artisti, committenti, collezionisti, Genova, Fondazione Carige, 2005, pp. 56-62.

iI. C. Spantigati, "Staziella carità sorger lo feo". Dipinti sculture e arredi tra antica e nuova sede, in C. Spantigati (a cura di), La Cattedrale di Alessandria, Alessandria, I988, p. I04 e tav. XII (la Madonna dell'Uscetto è già stata avvicinata all'anonimo Maestro della Madonna di Tersatto: G. Gamulin, Il Maestro della Madonna di Tersatto, in "Arte Veneta», $\mathrm{n}^{\circ}$ 34, 1980, pp. I8-25). Per la Madonna di Cambrai vedi Byzance. L'art byzantin dans les collections publiques françaises, catalogo della mostra, Parigi, 1992, pp. 478-479, scheda 369, di J. Durand e M. Rutschkowsky. Per il Maestro del dittico Sterbini cfr. E. B. Garrison, Italian Romanesque Panel Painting. An Illustrated Index, Firenze, Olschki, I949, p. II e n 65, 92, Io6 e 247 (il n 106 è di altra mano), da integrare con F. Zeri, La collezione Federico Mason Perkins, Torino, U. Allemandi \& C., i988, p. 82, e F. Zeri e F. Campagna Cicala, Messina, Museo Regionale, Palermo, Novecento, i992, pp. in e 55-56. Saltano agli occhi le affinità tra i Santi nel pannello sinistro del dittico Sterbini e gli affreschi del presunto Marco Greco sulla controfacciata del Duomo di Genova (G. Algeri, Tra Siena e Costantinopoli: $i$ nuovi modelli figurativi, in G. Algeri e A. De Floriani (a cura di), La pittura in Liguria. Il Medioevo, Genova, De Ferrari, 20II, pp. I4O-I43) e mostra qualche rapporto con il Maestro del dittico Sterbini anche la imponente Madonna col Bambino nella chiesa di Nostra Signora del Ponte a Lavagna, che iconograficamente deriva, con qualche variante di modernizzazione, dal modello "affettuoso» delle icone di Alessandria e di Cambrai: G. Algeri, Una tavola senese di primo Trecento nel Levante ligure, in «Bollettino d'Arte», 2009, I, pp. I-I2. La Madonna di Lavagna è stata restituita da ultimo al Maestro della Madonna di Santa Maria di Castello da Andrea de Marchi, credo a ragione: A Dominican Polyptych by the Master of the Piani d'Invrea Cross, Parigi, s.d. (ma 20I2), p. 20. È purtroppo tardo l'arrivo a Genova della bella Madonna bizantina «di Pera», già nell'oratorio di Sant'Antonio di Prè e ora al Museo di Sant'Agostino: L. Tagliaferro, L'Eleusa di Pera a Genova, in «Bollettino dei Musei Civici genovesi», XI, 1984, n I6-I8, pp. 7-23.

I2. Frater Thomas De Augustinis, Elenchica Synopsis... Conventus Divi Dominici Januae, manoscritto portato a termine nel I678, Genova, Biblioteca Universitaria (B. VIII. 4), pp. Io9-IIo.

I3. C. Di Fabio, Scultura romanica a Genova, Genova, Museo di Sant'Agostino, 1984, p. I49, n. Io.

I4. L. T. Belgrano, Anticaglie, in "Giornale ligustico di archeologia, storia e letteratura", giugno I886, p. 215. Non è detto che queste preziosità provenissero tutte dal tesoro di Pera portato a Genova nel I46I. 


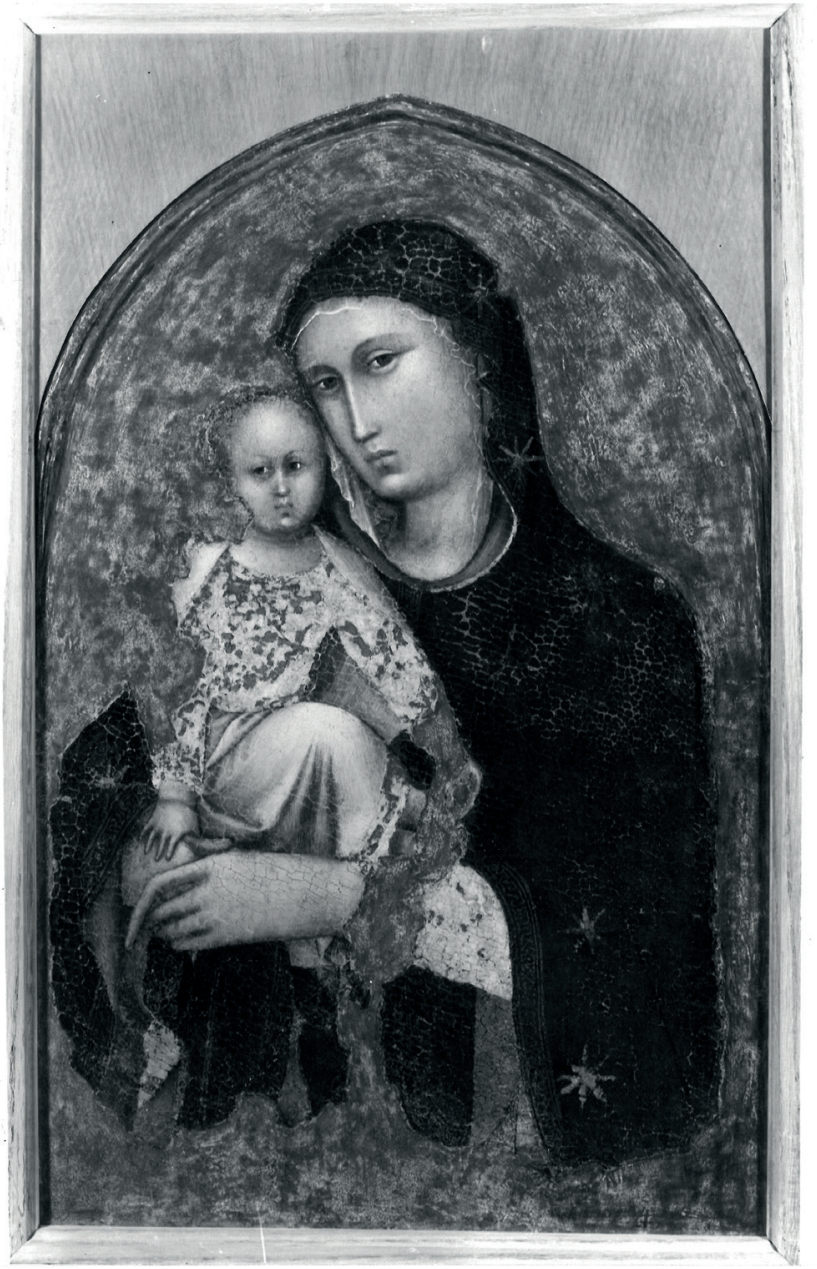

Fig. I. - Barnaba da Modena, Madonna con il Bambino. Lerma (Alessandria), Parrocchiale.

Foto Soprintendenza per i Beni Artistici e Storici, Torino.

Dobbiamo immaginare il primo Trecento ligure, come un campo di confronto e di scontro tra le novità assisiati e giottesche (via Pisa, come per la scultura), riconoscibili a partire dal Maestro della Madonna di Santa Maria di Castello ${ }^{15}$, e un persistente apprezzamento per i modelli orientali,

I5. F. Bologna, Alle origini della pittura ligure del Trecento. Il Maestro di Santa Maria di Castello e Opizzino da Camogli, in Hommage à Michel Laclotte. Études sur la peinture du Moyen Âge et de la Renaissance, Milano, Electa e Parigi, Réunion des musées nationaux, 1994, pp. 15-29. 
che arriva a determinare certe inceppature di disegno del polittico di Albi (un tempo a Lavagnola), che è del $1345^{16}$, o di un maestro attivo contemporaneamente a Barnaba come Francesco di Oberto. Per il Maestro del polittico di Albi sarebbe disponibile il nome storico di Giovanni Re, ma importa per il momento domandarsi se non gli si debba restituire la Madonna col Bambino del Museo di Chiavari (proveniente da Moneglia), parte centrale di un dossale all'antica simile a quello di cui ha fatto parte proprio la Madonna di Santa Maria di Castello ${ }^{17}$. Su una posizione più moderna si collocano il Maestro della Croce dei Piani di Invrea, il Maestro della Madonna di Tellaro, ultime importanti acquisizioni al panorama del primo Trecento ligure, e Bartolomeo da Camogli, che data al 1346 la Madonna dell'umiltà di Palermo ${ }^{18}$. Non ho dubbi sulla pertinenza ligure dal Maestro di Tellaro e del suo superbo capolavoro con la Flagellazione di Cristo ora a Malta, mentre ho molte perplessità sull'aggancio di Bartolomeo da Camogli alla cultura figurativa affermatasi con Simone Martini e Matteo Giovannetti ad Avignone (un'ipotesi che risale a Longhi e che Laura aveva tenuto in conto) ${ }^{\mathrm{I}}$, tanto più che il successo europeo dei modelli avignonesi non segue i percorsi della contiguità territoriale, ma piuttosto si rilancia a distanza, da corte a corte, fino all'inizio del secolo successivo. Trovo singolare che l'incorniciatura architettonica della Madonna di Palermo sia di gusto ancora romano-assisiate e che il tipo iconografico della Madonna dell'umiltà, con il Bambino al seno, non sia quello di Simone Martini ad Avignone, bensì quello, più arcaico, noto e replicato a Napoli, a Pisa e in altre regioni italiane. Mi sembra insomma

16. A. De Floriani, Rapporti tra la pittura della Francia meridionale e della Liguria tra XIV e XV secolo: alcune testimonianze significative, in M. Natale e S. Romano (a cura di), Entre l'Empire et la mer. Traditions locales et échanges artistiques (Moyen Âge - Renaissance), Actes du colloque de $3^{\mathrm{e}}$ Cycle Romand de Lettres, Lausanne - Genève, 22-23 mars, I9-20 avril, 24-25 mai 2002, Roma, 2007, pp. 75-93. Qualche debito verso la cultura figurativa bizantina era già stato avvertito, nel polittico di Lavagnola, da F. Alizeri, Notizie dei professori del disegno in Liguria dalle origini al secolo XVI, vol. I, Genova, I870, p. IoI: «del greco [stile] non v'ha che un'ombra lontana, e questa nel solo scomparto del Transito e più tosto nei canoni del significare la scena che nell'arte di esternare gli affetti». Sulla situazione genovese all'inizio del Trecento si vedano ora le importanti novità rese note da A. De Marchi, A Dominican Polyptych, citato a nota II.

17. G. Algeri (a cura di), Il Museo diocesano di Chiavari, Genova, Sagep, 1986, pp. 17-I8. Si attende Ead., L'immagine trecentesca della Madonna "dei Disciplinanti», in L'Oratorio dei Disciplinanti di Moneglia. Testimonianza di fede e di arte nella storia di una comunità, Atti del convegno, Moneglia IO-II ottobre 2008, in corso di stampa.

18. A. De Floriani, Bartolomeo da Camogli, Genova, Officina Grafica della Sagep S. p. A., i979; A. De Marchi, in Italies. Peintures des musées de la région Centre, catalogo della mostra a Tours, Orléans e Chartres, s.l., Somogy, Association des musées de la région Centre, 1996, pp. 45-5I (per il Maestro dei Piani di Invrea già identificato da Luciano Bellosi); ID., in P. Donati (a cura di), Restauri nel Golfo dei poeti, Genova, Sagep, 200I, pp. $70-78$ (per il Maestro di Tellaro). Riserve sul corpus del Maestro di Tellaro in A. De Floriani, Il fascino di Avignone, in G. Algeri e A. De Floriani (a cura di), La pittura in Liguria, cit., p. 204, nota 54. 19. L. Malvano, Barnaba da Modena, cit., p. 44. 
meno dispendioso credere all'esistenza di una precoce Madonna dell'umiltà martiniana a Napoli o a Pisa (forse meglio a Pisa), di notevole fortuna lungo le coste tirreniche ${ }^{20}$. I rapporti tra la cultura figurativa ligure e il ricco emporio martiniano di Pisa attendono di essere precisati, con attenzione particolare alla cronologia, magari per sostituire all'improbabile modello della Camera del Guardaroba avignonese (1343-1344) il ben più accessibile e qualificato polittico domenicano di Traini a Pisa, con la data del I345. Non è poi escluso che in Liguria si avesse notizia anche di personalità itineranti su percorsi defilati come "Antonius magister», alias Maestro della Incoronazione di Bellpuig, o il sorprendente e precoce Maestro del Crocifisso del cardinale Godin a Tolosa ${ }^{21}$.

Dalla tesi di Laura ad oggi è trascorso oltre mezzo secolo e il corpus di Barnaba si è arricchito di alcuni inediti, che non sembrano però aver inciso più di tanto nella ridefinizione della sua figura (a parte la Madonna di Lerma ovviamente). Laura stessa ha contribuito a queste aggiunte pubblicando una bella Madonna del Louvre (fig. 2), nel 1969 (RF 1968-4, da una collezione della regione di Nantes), e in quella occasione ha brevemente riconsiderato la cronologia del dipinti di Barnaba, di particolare difficoltà data la costanza tipologica delle sue Madonne ${ }^{22}$. Laura fissa intorno al 1375 la data della Madonna parigina, e il suo ragionamento si appoggia su una logica ricostruzione stilistica del percorso del pittore; ma che il problema cronologico sia ancora oggi di non concorde soluzione è dimostrato dal recente tentativo di fissare l'esecuzione del polittico di Ripoli tra il 1356 e il 1370 , con preferenza per una data alta $^{23}$. Credo che l'ipotesi non sia da

20. B. Williamson, The Madonna of Humility: Development, Dissemination and Reception, c. I340-I40o, Woodbridge, Boydell, 2009. È da rilevare che nessuna delle illustrazioni del volume documenti una effettiva fortuna iconografica della Madonna di Simone Martini ad Avignone: ha successo solo il tipo della Virgo lactans.

2I. F. Bologna, Di alcuni rapporti tra Italia e Spagna nel Trecento e "Antonius magister", in "Arte antica e moderna", 196I (Raccolta di saggi dedicati a Roberto Longhi in occasione del suo settantesimo compleanno), pp. 27-48; M. Bosкoviтs, Il problema di "Antonius magister» e qualche osservazione sulla pittura marchigiana del Trecento, in "Arte illustrata», II, I969, $\mathrm{n}^{\circ}$ I7-I8, pp. 4-I9; M. L. Testi CRistiani, "Circostanze avignonesi». Il Crocifisso double-face del cardinale Godin a Tolosa, I, in "Critica d'Arte», ottobre-dicembre I990, pp. 42-6I.

22. L. Malvano, Une Vierge à l'Enfant de Barnaba da Modena au Louvre, in «La Revue du Louvre et des Musées de France», XIX, I969, n 6, pp. 339-346. Cha Laura intendesse continuare a lavorare su Barnaba è dimostrato dalla presenza di molte nuove fotografie, oltre a quelle usate per la tesi, nel suo dossier di lavoro che l'amico Antonio Bechelloni mi ha cortesemente messo a disposizione. Tra le altre sono da segnalare due diverse riprese della Madonna "homeless» pubblicata da B. Berenson, Italian Pictures of the Renaissance. Central Italian and North Italian Schools, Londra, Phaidon, 1968, vol. I, p. 29; vol. II, fig. 274. È inedita la ripresa più antica, con la tavola, terminante a profilo cuspidato, incastonata in una ricca cornice a tabernacolo.

23. P. Carofano, Il polittico di Barnaba da Modena nella Parrocchiale di Ripoli, in "Quaderni cascinesi», V/I, dicembre I990, pp. 36-47. Qualche chiarezza sulla cronologia delle opere di Barnaba è venuta dall'accertamento che non è affidabile la data 1352 sulla Santa Caterina ora a Palazzo Spinola di Genova: F. SimonetTi e G. Zanelli (a cura di), La Santa Caterina di Barnaba da Modena, Roma, Novamusa, 2005. Non meno 
accogliere e che convenga meglio una data tarda, tanto più che la Madonna di Ripoli si avvicina per stile e per tipo iconografico proprio alla Madonna del Louvre. Laura non è più tornata sull'argomento della tesi nonostante avesse promesso un intervento sui polittici di Murcia, che aveva voluto studiare da vicino. Per parte mia ho tentato inutilmente di sollecitarla procurandole la fotografia della quasi sconosciuta predella di Kiev $\left(\mathrm{n}^{\circ} 493\right.$, $\mathrm{cm} 43,5 \times 2$ IO) che, fatti salvi i riferimenti dei cataloghi russi, ha goduto finora di una sola fuggevole citazione di Laclotte nel breve saggio sulla Crocifissione di Caen ${ }^{24}$. Sul filo degli assestamenti cronologici e del reperimento di inediti si sono posti gli interventi più recenti sul pittore, in particolare quelli della Algeri, che non sono però riusciti ad eliminare dal corpus di Barnaba alcuni dipinti non pertinenti: un trittico certamente non ligure delle Collezioni Comunali di Bologna ${ }^{25}$, la Madonna col Bambino passata alla Finarte di Roma il I4 novembre 2006 (asta n ${ }^{\circ}$ I353, lotto 2), che sospetto falsa ${ }^{26}$, la Madonna frammentaria di Santa Maria di Castello a Genova, già inserita in una tela di Aurelio Lomi ${ }^{27}$; nonostante l'ampiezza dei danni questa tavola va restituita al bel Maestro della Madonna del Bosco nella Cattedrale di Sassari, per come lo conosciamo dopo la liberazione del dipinto sassarese dalle ridipinture quattrocentesche, che però temo fossero di mano del Maestro di Castelsardo. Ho qualche difficoltà anche ad accogliere la Crocefissione di Caen che mi arrischierei ad accostare al corpus pittorico di Guglielmetto Fantini. È invece una bella e positiva novità la Santa Caterina d'Alessandria (fig. 3) fatta conoscere dal compianto Miklos Boskovits nel suo ultimo intervento genovese (I4 giugno 20II, per la presentazione del volume La pittura in Liguria. Il Medioevo, di Algeri e De Floriani) e mi provo anch'io a suggerire alla discussione degli appassionati di Barnaba alcune proposte un poco fuori contesto. Presso l'altare di san Giuseppe nella Jesuitenkirche di Vienna è esposta una Madonna col Bambino, di formato ovale, dai caratteri di stile

rivelatore è stato il restauro dei polittici di Murcia, difficilmente riferibili a un momento giovanile della carriera del pittore: A. E. Pérez SÁnchez, Las obras restauradas, in Obras maestras restauradas. Fondación Argentaria, Madrid, Fundación Argentaria, 1993, pp. 16-28.

24. M. Laclotte, Un panneau de Barnaba da Modena, in "La Revue du Louvre et des Musées de France», XIV, I964, n ${ }^{\mathrm{i}} 4-5$, p. I80, n. 8.

25. G. Algeri, L'attività tarda di Barnaba da Modena: una nuova ipotesi di ricostruzione, in "Arte Cristiana", 1989, $\mathrm{n}^{\circ}$ 732, p. 204, n. 23 (la Algeri non tiene conto della scheda di Carlo Volpe in Pelagio Palagi artista e collezionista, catalogo della mostra, Bologna, Biblioteca comunale dell'Archiginnasio, 1976, p. 424, $\mathrm{n}^{\circ} 545$ ).

26. G. Algeri, L'attività di Barnaba da Modena, in G. Algeri e A. De Floriani (a cura di), La pittura in Liguria, cit., p. 208. È a suo modo significativo che nel 1939 la piccola tavola ( $\mathrm{cm} 32$ x 2I) sia stata attribuita privatamente a Lippo Memmi da un conoscitore di cose liguri come William Suida.

27. G. Rotondi Terminiello, in Restauri in Liguria, catalogo della mostra, Genova, I978, scheda I3, pp. $235-240$. 
indubbiamente alla Barnaba: la redazione pittorica lascia qualche dubbio e del resto una tradizione di sacrestia vuole che sia copia di una immagine miracolosa conservata in un santuario peruviano (Madonna di Uzategui). Non è da escludere che qualche originale di Barnaba sia emigrato oltreoceano come immagine di maniera greca particolarmente miracolosa e si potrebbe anche sospettare che Barnaba stesso abbia eseguito, secondo una abitudine bolognese, ingannevoli icone all'antica. Ha sicuramente avuto in origine un ruolo di immagine miracolosa la vivace Madonna (fig. 4) vicinissima a Barnaba della vecchia collezione Acton di Firenze, documentata da due diverse fotografie dello studio Jacquier ${ }^{28}$. Mi domando infine se non sia da ricondurre ai quesiti riguardanti Barnaba anche il polittico di Sant'Orsola, dalla chiesa dei Santi Giacomo e Filippo, ora nei depositi del Museo di Sant'Agostino a Genova ${ }^{29}$. Contrariamente a quanto sembra far credere la scritta ai piedi della santa la carpenteria non è cinquecentesca, bensì di due secoli prima, come confermano i piccoli campioni di pulitura sulla cornice che hanno rivelato una bella decorazione trecentesca a girali vegetali dorati in foglia. Anche le storiette ai lati conservano con buona fedeltà, nonostante le replicate ridipinture integrali, dei partiti iconografici non troppo dissimili (se mai più ricchi) di quelli riconoscibili nei polittici di Barnaba al Museo diocesano di Genova e nella Cattedrale di Murcia. C’è insomma ancora da fare intorno al pittore amato dalla giovane Laura.

28. M. Tamassia, Collezioni d'arte tra Ottocento e Novecento. Jacquier fotografi a Firenze I870-I935, Napoli, Electa, I995, p. 2I.

29. Vedi L. Tagliaferro (a cura di), Il dipinto e il suo rovescio, catalogo della mostra, Genova, I99I, p. 25. Ringrazio Piero Boccardo che, insieme ad Adelmo Taddei, ha agevolato il mio sopralluogo nei depositi del Museo di Sant'Agostino. 


\section{Giovanni Romano}

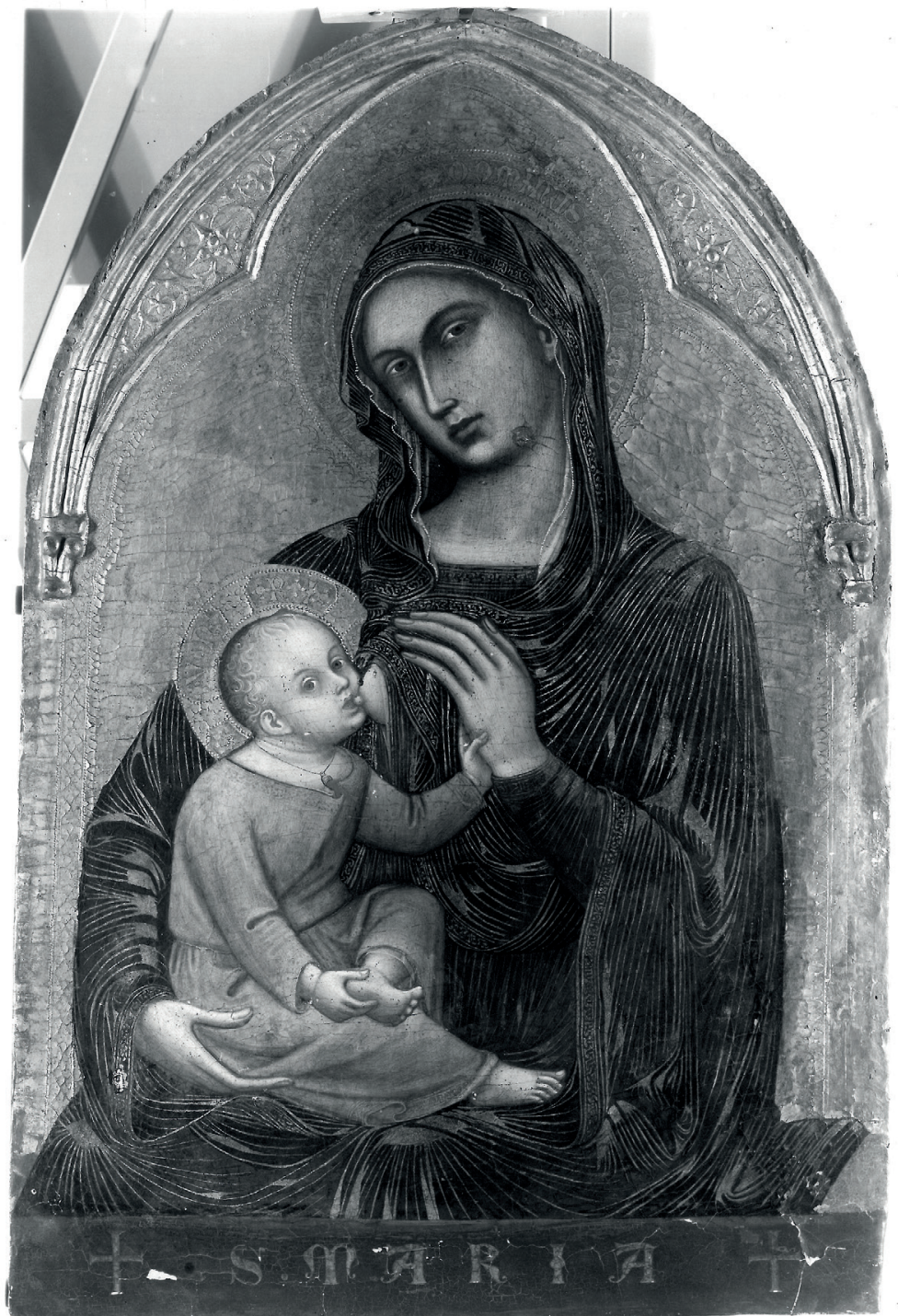

Fig. 2. - Barnaba da Modena, Madonna con il Bambino. Parigi, Louvre.

Foto Institut Mainini. Laboratoire du Musée du Louvre, Parigi. 


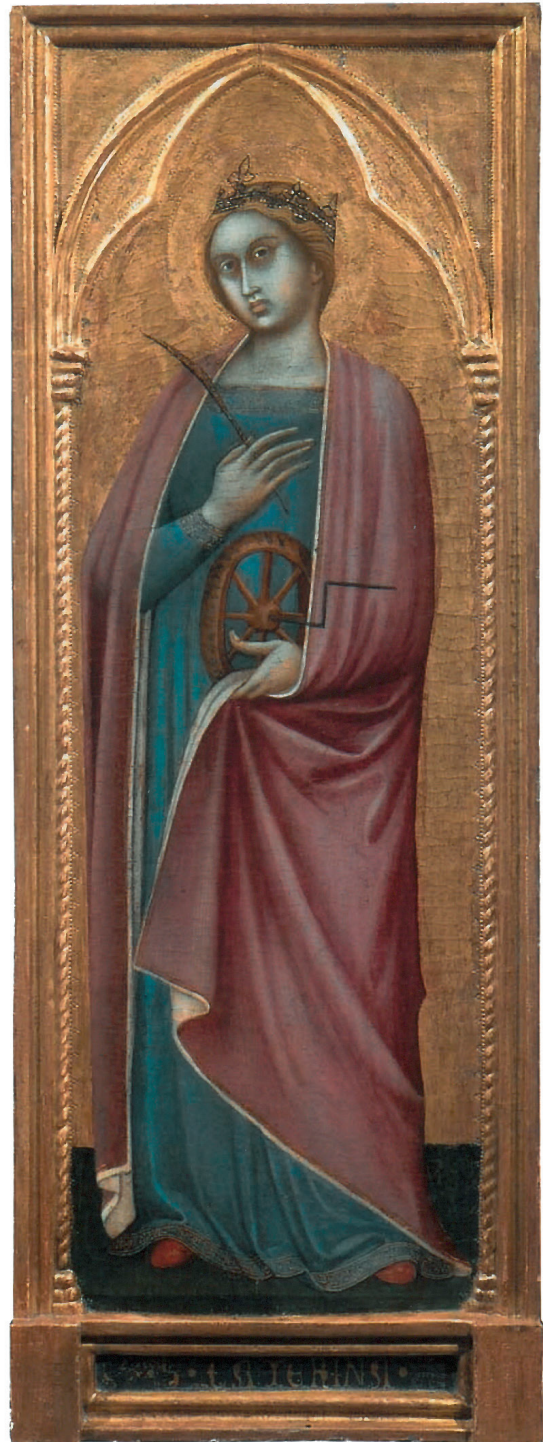

Fig. 3. - Barnaba da Modena, Santa Caterina d'Alessandria. Mercato antiquario.

Foto gentilmente concessa da Miklos Boskovits. 


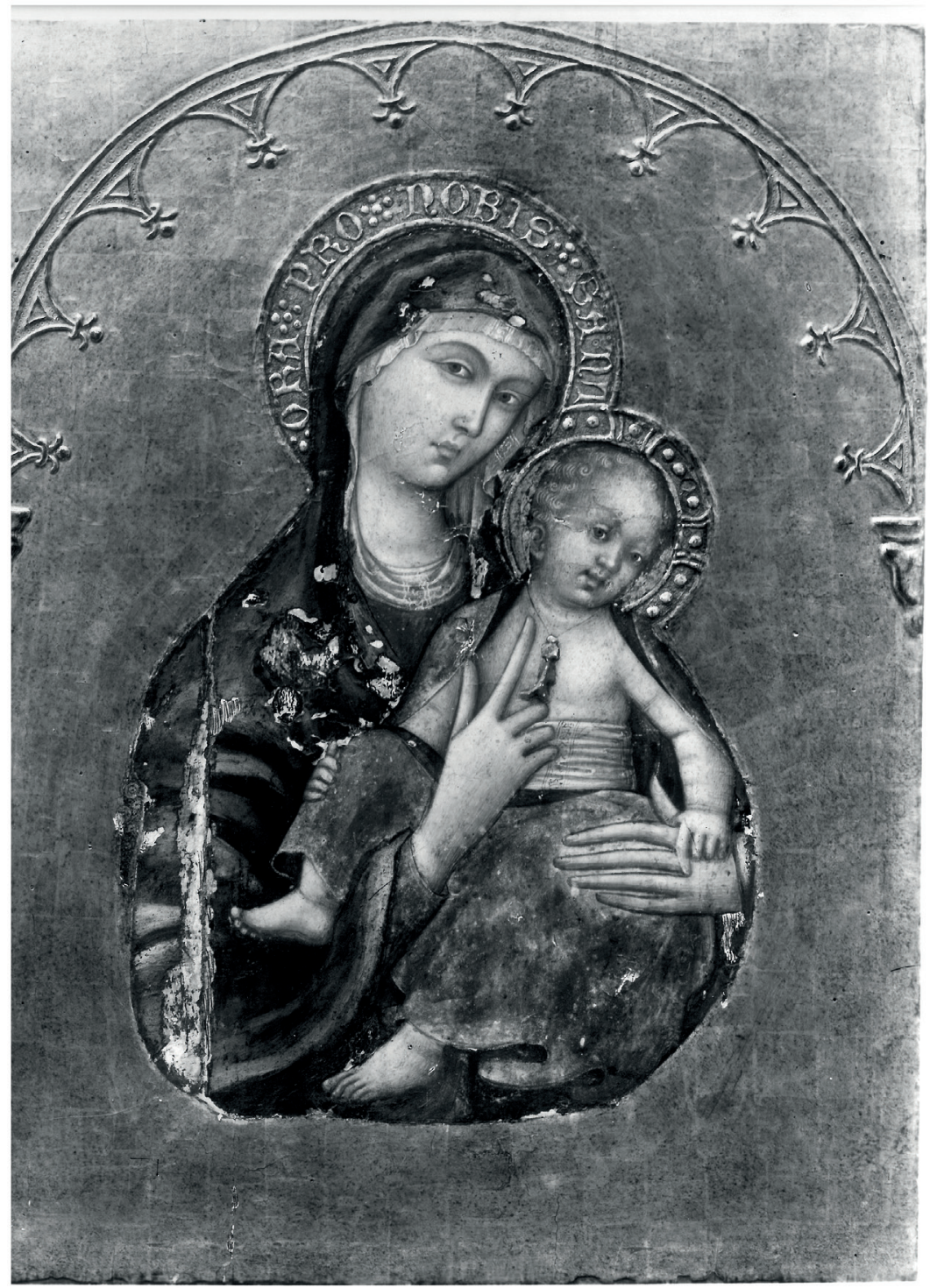

Fig. 4. - Pittore affine a Barnaba da Modena, Madonna con il Bambino. Firenze, già collezione Acton.

Foto Soprintendenza per i Beni Artistici e Storici, Firenze (archivio Jacquier). 